\title{
El Papel de la Cooperación para Desarrollar Innovación Tecnológica en la PYME
}

\author{
Mauricio Castillo-Vergara ${ }^{*^{*}}$ Enrique Torres Aranibar ${ }^{2}$
}

Resumen: La innovación se ha consolidado como una vía para mejorar el desempeño de las economías. El objetivo del estudio es analizar el rol de las cooperaciones y los efectos en la innovación tecnológica y el desempeño de las empresas aplicando PLS-SEM. Para probar el modelo se utilizó la décima encuesta de innovación del Ministerio de Economía de Chile, levantada durante el año 2017. Los resultados muestran que la cooperación comercial y cooperación profesional tienen efecto en la innovación tecnológica, sin embargo, la cooperación académica no produce efecto significativo. Se han identificado acciones que permitan mejorar el sistema de innovación.

Palabras Claves: Innovación; Cooperación; Desempeño; Economía Emergente; PYME.

The role of cooperation to develop technological innovation in SMEs

Abstract: Innovation has established itself to improve the performance of economies. The objective of the study is to analyze the role of cooperation's and the effects on technological innovation and the performance of companies applying PLS-SEM. To test the model, the tenth innovation survey of the Ministry of Economy of Chile, carried out during 2017, was used. The results show that commercial cooperation and professional cooperation influence technological innovation, however, academic cooperation has no significant effect. Actions that improve the innovation system have been identified.

Keywords: Innovation; Cooperation; Performance; Emerging Economy; SME

Submitted: March 27 $7^{\text {th }}, 2019$ / Approved: November $4^{\text {th }}, 2019$

\section{Introducción}

La innovación es una vía para generar desarrollo económico y crecimiento (Tello Gamarra, Machado Leo, Silva Avila, \& Wendland, 2019) y ocupa un lugar destacado en la agenda estratégica de la mayoría de las economías (Casanova, Cornelius, \& Dutta, 2017). Un factor importante dentro de la supervivencia económica de una empresa es el desarrollo de una ventaja competitiva (Lafuente, Leiva, Moreno-Gómez, \& Szerb, 2019), la cual puede potenciarse mediante la innovación (Höflinger, Nagel, \& Sandner, 2017).

Para fomentar la innovación dentro de las empresas se requiere adquirir conocimientos (Scandura, 2016), de hecho, parte del proceso de innovación incluye el intercambio de información, la resolución de problemas y el aprendizaje entre personas u organizaciones heterogéneas (Edwards-Schachter, 2018). Para innovar con éxito, las empresas deben participar cooperativamente para acceder a recursos y capacidades con otras entidades (Heidenreich, Landsperger, \& Spieth, 2016). Esta acción es necesaria para crear nuevos productos, procesos y tecnologías, especialmente en economías emergentes, donde los recursos y capacidades de innovación son relativamente limitados (Rojas, Solis, \& Zhu, 2018). Se afirma que la innovación no ocurre aislada, sino destacando el papel de las interacciones entre organizaciones, el gobierno y las instituciones de investigación (Tello Gamarra et al., 2019) y y existe un llamado para estudiar el impacto de las actividades innovadoras en el desempeño de la empresa (Hashi \& Nebojsa, 2013).
A pesar de la creciente investigación sobre innovación, existen una brecha que la literatura ha explorado insuficientemente y que este artículo intenta explicar. Los estudios en el contexto de la pequeña y mediana empresa (PYME) ha recibido poca atención (Abdul-Halim, Ahmad, Geare, \& Thurasamy, 2019; Lavoie, Lavoie, Abdulnour, Lavoie, \& Abdulnour, 2015), en particular en países emergentes (Heredia Pérez, Geldes, Kunc, \& Flores, 2019) y el conocimiento que se ha desarrollado sobre prácticas de innovación en grandes empresas no es fácilmente transferible a la PYME (Radziwon \& Bogers, 2018).

Durante la última década, el gobierno de Chile ha impulsado medidas para mejorar el sistema nacional de innovación (Guimón, Chaminade, Maggi, \& Salazar-Elena, 2018), duplicando el presupuesto para proyectos de innovación (Heredia, Geldes, Kunc, \& Flores, 2019). En Chile. cifras de la Corporación de Fomento de la Producción (CORFO) indican que el presupuesto destinado a proyectos de innovación ha aumentado en un $200 \%$, con una cobertura de más de 270.000 beneficiarios durante el período 2009 - 2013 (CORFO, 2013). Sin embargo, según la última encuesta de innovación sólo el 15,1\% de las empresas Chilenas ha innovado (Instituto Nacional de Estadisticas, 2018), situación que comparte con otros países latinoamericanos y emergentes (Geldes \& Felzensztein, 2013). Con lo anterior, Chile representa un entorno interesante para los fines de la investigación. Abordar la relación entre cooperación e innovación resulta interesante para las PYMES (Perkins, Lean, \& Newbery, 2017, y estas se pueden ver favorecidas, ya que el desarrollo de innovación es considerado un desafío central de las empresas (Sarooghi, Libaers, \& Burkemper, 2015).

(1) Facultad de Economía y Negocios, Universidad Alberto Hurtado, Santiago de Chile.

(2) Departamento de Ingeniería Industrial, Universidad de La Serena, La Serena, Chile.

*Autor de correspondencia: mhcastillo@uahurtado.cl 
Para innovar con éxito, las empresas deben participar en la cooperación para obtener acceso a recursos y capacidades de otras entidades (Heidenreich et al., 2016), aún cuando, un cuerpo de investigación se ha ocupado de la cooperación en general, la investigación sobre cooperación que apunta específicamente a fomentar la innovación ha recibido menos atención (Weber \& Heidenreich, 2018) y parece de suma importancia saber que tipo de cooperación es la más efectiva para mejorar el resultado de la innovación, pero los estudios sobre los efectos de la cooperación en el desempeño de la innovación son escasos (Park, Srivastava, \& Gnyawali, 2014).

Esta noción de cooperación sugiere que los actores no son independientes ni autosuficientes y que necesitan interactuar con otros para obtener beneficios, como adquirir conocimiento y recursos para la innovación (Chou \& Zolkiewski, 2018). La cooperación puede existir en muchos niveles diferentes, entre individuos, equipos y departamentos, organizaciones y redes (Lindström \& Polsa, 2016), en este documento consideramos la cooperación de forma sistémica al explorar los diferentes tipos de cooperación: cooperación académica
(Universidades, Institutos de Investigación y Artículos Científicos), cooperación comercial (Clientes, Proveedores, Competidores y Consultores) y cooperación profesional (Profesional e Industrial, Conferencias, Ferias o Exposiciones y Profesionales al interior de las organizaciones).

El documento está organizado de la siguiente manera. En la siguiente sección, se presentan el modelo y las hipótesis a evaluar. Los datos y métodos utilizados para probar estas hipótesis se discuten en la sección metodología. En resultados y discusión se informan y discuten los resultados. Finalmente, se identifican conclusiones, limitaciones y propuestas de investigación futura.

\section{Modelo}

El modelo de estudio propuesto se estructura como se indica en la figura 1, que señala la existencia de seis constructos. Para implementar empíricamente el modelo se utilizan ecuaciones estructurales basadas en mínimos cuadrados parciales (PLS) y se aplica el uso del software SmartPLS versión 3.2.8 (Ringle, Wende, \& Becker, 2017).

Figura 1. Conjunto de relaciones descritas en las hipótesis

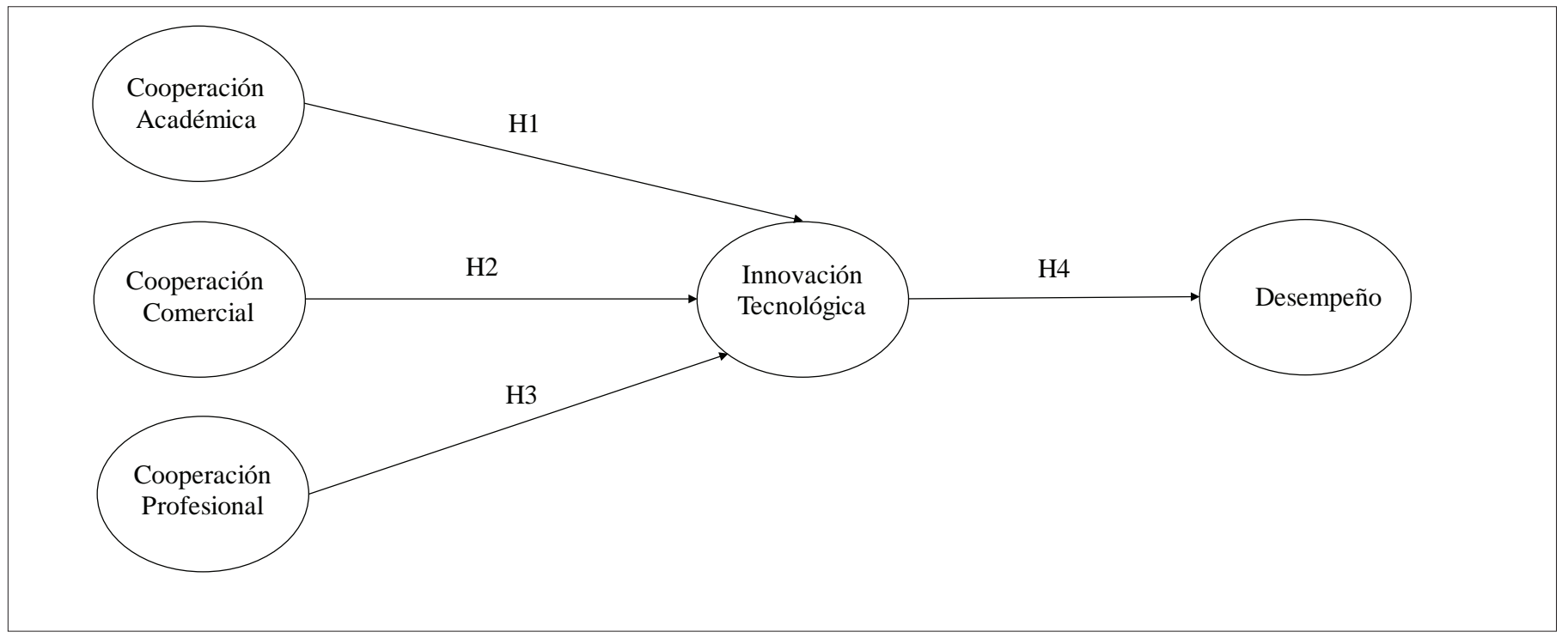

Fuente: Elaboración Propia

\section{Cooperación académica e innovación tecnológica}

Zeng, Xie y Tam (2010) mencionan que las universidades y los institutos de investigación tienen un impacto valioso dentro del proceso de creación de conocimiento e innovación y generalmente ocurren mediante un intercambio explícito de conocimiento (Ben Arfi, Hikkerova, \& Sahut, 2018). Este tipo de cooperación es importante para empresas sin experiencia en I+D interna e incluso para aquellas que desean potenciar sus capacidades internas de innovación (Serrano-Bedia, López-Fernández, \& García-Piqueres, 2018). Las fuentes externas de conocimiento pueden agruparse en tres categorías: universidades, institutos de investigación y empresas intensivas en conocimiento (Vivas \& Barge-Gil, 2015).
La colaboración entre la universidad y la industria para I+D e innovación ha ido en aumento los últimos años debido a la combinación de estímulos y presiones tanto en la industria (cambios tecnológicos, globalización, ciclos de vida del producto más cortos y tiempo de comercialización, etc.) como en las universidades (financiación externa, políticas gubernamentales, etc.) (Giannopoulou, Barlatier, \& Pénin, 2019). Por otro lado, los institutos de investigación públicos son uno de los actores más importantes dentro de los sistemas nacionales de innovación (Intarakumnerd \& Goto, 2018). Los beneficios de la cooperación académica dentro del proceso de innovación son diversos, como por ejemplo optar a equipos de laboratorio, compartir riesgos y adquirir conocimientos complementarios (Lin, 2017), es por esto que se plantea la siguiente hipótesis: 


\section{H1: La cooperación académica afecta positivamente la innovación tecnológica}

\section{Cooperación comercial e innovación tecnológica}

Desde la perspectiva de la competencia, se espera que las empresas que cooperan obtengan más beneficios de innovación y aprovechen en mayor medida los activos de las alianzas (Park et al., 2014). Sin embargo, cooperar con los competidores no es la única forma en que una empresa adquiere y desarrolla capacidades de innovación de productos, existe una tendencia creciente a aplicar la cooperación entre los socios de la cadena de suministro como una estrategia eficiente para crear valor (Resende et al., 2018). A nivel comercial, algunas contribuciones han tratado con empresas que cooperan con competidores, proveedores, clientes (Dorn, Schweiger, \& Albers, 2016). En el contexto de la PYME se apunta a ganar poder de negociación mediante la cooperación con competidores (Schiavone \& Simoni, 2011) e ingresan de mejor manera en algunos mercados colaborando con competidores o clientes (Akdoğan \& Cingšz, 2012). La cooperación incluye varios jugadores, como los clientes, proveedores, complementadores y socios (Bengtsson \& Raza-Ullah, 2016) y puede promover la innovación a través de la competencia (Gnyawali \& Park, 2011). Académicos sostienen que la cooperación mejora el intercambio de información, ayuda a obtener economías de escala, reduce la incertidumbre y los riesgos y acelera el desarrollo de nuevos productos ( $\mathrm{Wu}$, 2014), dado que las actividades de las empresas están relacionadas con actividades y conocimiento de una serie de contrapartes. (Chou \& Zolkiewski, 2018). Con estos antecedentes se plantea la siguiente hipótesis:

\section{H2: La cooperación interempresarial afecta positivamente la innovación tecnológica}

\section{Cooperación profesional e innovación tecnológica}

La cooperación profesional son para las organizaciones el medio por el cual pueden acceder a fuentes de información externa, que son necesarias para involucrarse en nuevos mercados (Bolívar-Cruz, Fernández-Monroy, \& Galván-Sánchez, 2017), y la cooperación simultáneas de diversas fuentes pueden facilitar la innovación y la creatividad dentro de los equipos (Dorn et al., 2016). Estos impulsores se relacionan con la empresa (Bengtsson \& Raza-Ullah, 2016) y se ha destacado la relevancia de las colaboraciones para la innovación en las empresas, incluyendo específicamente varios contactos profesionales (Trigo, 2011), con lo cual la cooperación favorece la generación y difusión de conocimiento e innovación (Bolívar-Cruz et al., 2017). Con lo anterior se plantea la siguiente hipótesis:

\section{H3: El desarrollo de I+D intramuro afecta positivamente la innovación tecnológica}

\section{Innovación tecnológica y desempeño}

La literatura relacionada con el éxito de la innovación tecnológica en el rendimiento de las empresas es extensa y ha sido respaldada en diversos sectores (Ferreira, Coelho, \& Moutinho, 2018; Genc, Dayan, \& Genc, 2019). Las innovaciones tecnológicas permiten responder rápidamente a las necesidades de los clientes, ganar participación de mercado, aumentar la calidad y variedad de los productos (Fossasolalla, Minguela-rata, \& Fernández-menéndez, 2015), una mayor propensión a innovar se asocia con un mejor desempeño del mercado de exportación y financiero (Hughes et al., 2018). La innovación también aparece como un motor crítico para el desempeño (Gunday, Ulusoy, Kilic, \& Alpkan, 2011). Ferreira et al. (2018) explica el crecimiento de una empresa a través de la innovación y tiene un efecto positivo en el rendimiento del negocio (Atalay, Anafarta, \& Sarvan, 2013; Crespell \& Hansen, 2008; Rajapathirana \& Hui, 2017) y en el rendimiento financiero (Karabulut, 2015). Estos hallazgos conducen a la siguiente hipótesis:H4: La innovación tecnológica afecta positivamente el desempeño de las empresas

\section{Metodología}

\section{Datos utilizados}

Para probar las hipótesis y el modelo, se ha utilizado la base de datos correspondiente a la "Décima Encuesta de Innovación en Empresas" del Ministerio de Economía de Chile, levantada durante el año 2017 tomando como años de referencia a 2015 y 2016 (Instituto Nacional de Estadisticas, 2018). El diseño y metodología siguen los lineamientos sugeridos por la (OECD/European Communities, 2005). Los datos corresponden a 5.876 empresas, de este conjunto de datos, se han seleccionado 3.252 empresas que corresponden a 2.048 empresas categorizadas en pequeñas (entre 11 y 50 trabajadores) y 1.474 empresas categorizadas en medianas (entre 51 y 250 trabajadores), según el número de trabajadores promedio entre los años 2015 y 2016, no se ha seleccionado ningún sector ni región en particular.

\section{Variables utilizadas}

La principal característica de la encuesta de innovación es que contiene un detalle de la introducción de los diferentes tipos de innovación y desempeño en las empresas. También incluye diferentes fuentes de cooperación en actividades innovativas, la confección de las variables se ha construido de acuerdo con el siguiente detalle:

Cooperación Académica: Importancia de aquellas fuentes de cooperación para el desarrollo de actividades innovativas en los años 2015 y/o 2016. Se ha utilizado la intensidad del tipo de cooperación empleada, medida como alta, media, baja o nula (Weber \& Heidenreich, 2018). Tanto por las universidades u otras instituciones de educación superior e institutos de investigación públicos. Adoptamos la propuesta de Szücs (2018), respecto a las colaboraciones académicas que incluyen universidades, así como colaboraciones con instituciones de investigación y revistas científicas, publicaciones técnicas y comerciales, y bases de datos de patentes.

Cooperación Profesional: Importancia de aquellas fuentes de cooperación para el desarrollo de actividades innovativas en los años 2015 y/o 2016. Se ha utilizado la intensidad del tipo de cooperación empleada, medida como alta, media, baja o nula (Weber \& Heidenreich, 2018). Las preguntas trataron los siguientes aspectos: Para las actividades innovativas la empresa utilizó en los años 2015 y/o 2016 señale la importancia de cooperaciones a nivel profesional e industrial, conferencias, ferias o exposiciones y profesionales al interior de las organizaciones. 
Cooperación Comercial: Importancia de aquellas fuentes de cooperación para el desarrollo de actividades innovativas en los años 2015 y/o 2016. Se ha utilizado la intensidad del tipo de cooperación empleada, medida como alta, media, baja o nula (Weber \& Heidenreich, 2018). Las preguntas trataron los siguientes aspectos: Para las actividades innovativas la empresa utilizó en los años 2015 y/o 2016 señale la importancia de cooperaciones con clientes, proveedores, competidores y consultores.

Innovación Tecnológica: Utilizamos la escala que distingue innovación de producto y proceso como indicadores dicotómicos, 0 para respuesta negativa y 1 si la empresa introdujo en los años 2015 y/o 2016 los siguientes aspectos: (1) Bienes nuevos o significativamente mejorados (excluye la simple reventa de productos nuevos comprados a otras empresas y los cambios de carácter exclusivamente estéti$\cos (2)$ Servicios nuevos o significativamente mejorados (3) Un nuevo o significativamente mejorado método de manufactura o producción de bienes o servicios (4) Una nueva o significativamente mejorada actividad de soporte para sus procesos, tales como sistema de mantención u operaciones de compras, contabilidad o informática.

Desempeño: Para la construcción del desempeño, se incluyen resultados del producto o proceso (Laforet, 2013) e incluye preguntas sobre la importancia de las innovaciones de productos y procesos (Gunday et al., 2011) en los años 2015 y/o 2016. Las preguntas trataron los siguientes aspectos: (1) Ampliación de la gama de bienes y servicios, (2) Ingreso a nuevos mercados o incrementos de la participación en el mercado actual, (3) Mejora en la calidad de los bienes y servicios, (4) Aumentar la capacidad y/o flexibilidad para la producción de bienes y servicios, (5) Reducción de costos por unidad producida y (6) Reducción de impacto medioambiental o mejorar la sanidad y la seguridad.

\section{Método de Análisis}

En las últimas décadas los modelos de ecuaciones estructurales (SEM) se han convertido en una importante herramienta del análisis multivariante y su uso se ha extendido en la investigación en ciencias sociales (Cepeda Carrión \& Roldán Salgueiro, 2004). Los SEM combinan el uso de variables no observadas (latentes) que representan conceptos teóricos y datos que provienen de medidas (indicadores o variables manifiestas), los que son usados como insumos para proporcionar evidencia sobre las relaciones entre las variables latentes (Williams, Vandenberg, \& Edwards, 2009). Los modelos valoran en un análisis único, el modelo de medida y el modelo estructural (Gefen, Straub, \& Boudreau, 2000). El análisis de los SEM puede ser llevado a cabo por dos tipos de técnicas: basados en el análisis de las covarianzas o basados en la varianza (Barroso, Cepeda Carrión, \& Roldán, 2010). Ambos enfoques han sido diseñados para objetivos distintos, mientras el método basado en el análisis de las covarianzas se enfoca en estimar un conjunto de parámetros del modelo para que la matriz de covarianza teórica implícita por el sistema de ecuaciones estructurales esté lo más cerca posible de la matriz de covarianza empírica observada dentro de la muestra de estimación (Reinartz, Haenlein, \&
Henseler, 2009), el método Partial Least Squares (PLS) utiliza bloques de variables (componentes) y estima los parámetros del modelo por medio de la maximización de la varianza explicada de todas las variables dependientes (incluidas latentes como observadas) (Chin, 1998). Partial Least Squares puede ser usado con múltiples propósitos, y es una técnica adecuada para fines de exploración, explicación o confirmación si un modelo de ecuación estructural contiene una o más construcciones operativas como un compuesto (Henseler, 2018). Como se indicó previamente, será utilizada la herramienta SmartPLS 3.2.8 (Hair, Hult, Ringle, \& Sarstedt, 2017). La técnica se ha seleccionado por presentar ciertas ventajas, no impone suposición de distribución específica (p.ej. normalidad) para los indicadores y no necesita que las observaciones sean independientes unas de otras (Chin, 2010), los requerimientos sobre escalas de medida son mínimos (Wold, 1984) y no requiere uniformidad en las escalas de medida (Sosik, Kahai, \& Piovoso, 2009).

El método PLS permite el uso de tres tipos de modelos de medida: formativos, reflectivos y modelos compuestos (Henseler, Ringle, \& Sarstedt, 2016), y su elección debe sustentarse en la naturaleza del constructo (Henseler, 2016). Siguiendo a (Xu, Peng, \& Prybutok, 2019) todos los indicadores de las variables en estudio se han analizado como modo compuesto tipo A.

\section{Resultados}

Tal como se ha señalado en este estudio se utiliza la técnica PLS, cuya aplicación consta de dos pasos: (1) La evaluación del modelo de medida y (2) la evaluación del modelo estructural (Barclay, Higgins, \& Thompson, 1995). Se estima el modelo estructural saturado aplicando un proceso de bootstrapping (5,000 submuestras) (Henseler, Hubona, \& Ray, 2016). El Residual Cuadrado Medio Residual Estandarizado $(\mathrm{SRMR}=0,055)$ está por debajo de 0,08 sugerido por ( $\mathrm{Hu} \&$ Bentler, 1999), con esto, se obtiene evidencia empírica para los constructos operacionalizados.

Siguiendo las recomendaciones de (Müller, Schuberth, \& Henseler, 2018) se debe establecer la confiabilidad de los indicadores y constructos, la validez convergente y validez discriminante. La carga $(\lambda)$ de cada elemento en su construcción debe ser superior a 0,707 para verificar la confiabilidad del indicador (Hair et al., 2017), aún cuando tres elementos se encuentran por debajo de 0,707 estos fueron retenidos según lo sugerido por (Martelo-Landroguez, Cegarra Navarro, \& Cepeda-Carrión, 2019). Para establecer la confiabilidad del constructo el coeficiente alfa de Cronbach debe ser superior a 0,8 para ser considerado excelente y sobre 0,65 para ser considerado aceptable (Castillo-Vergara, Barrios Galleguillos, Jofré Cuello, Alvarez-Marin, \& Acuña-Opazo, 2018), la fiabilidad compuesta y el indicador Dijkstra-Henseler's (rhoA) deben ser superior a 0,7 (Hair et al., 2017) y para establecer la validez convergente los valores deben ser mayores a 0,5 (Fornell \& Larcker, 1981). En la tabla 1 se presentan los resultados para cada uno de los constructos, estos resultados indican que los modelos de medición son internamente consistentes y confiables y se obtiene una validez convergente aceptable. 
Tabla 1. Resultados para la evaluación de medidas

\begin{tabular}{|c|c|c|c|c|c|c|}
\hline Constructo & Ítems & $\begin{array}{c}\text { Carga } \\
(\lambda)\end{array}$ & Fiabilidad Compuesta & $\begin{array}{l}\text { Alpha de } \\
\text { Cronbach }\end{array}$ & rho A & AVE \\
\hline \multirow[t]{2}{*}{ Cooperación Académica } & ACA1 & 0,799 & 0,819 & 0,682 & 0,708 & 0,602 \\
\hline & ACA3 & 0,811 & & & & \\
\hline \multirow[t]{2}{*}{ Cooperación Profesional } & PRO1 & 0,810 & 0,812 & 0,663 & 0,708 & 0,592 \\
\hline & PRO3 & 0,690 & & & & \\
\hline \multirow[t]{3}{*}{ Cooperación Comercial } & COM1 & 0,818 & 0,836 & 0,740 & 0,762 & 0,563 \\
\hline & COM2 & 0,804 & & & & \\
\hline & COM3 & 0,730 & & & & \\
\hline \multirow{2}{*}{ Innovación Tecnológica } & IT3 & 0,724 & & & & \\
\hline & IT4 & 0,685 & & & & \\
\hline \multirow[t]{6}{*}{ Desempeño } & EF1 & 0,942 & 0,980 & 0,976 & 0,708 & 0,891 \\
\hline & $\mathrm{EF} 2$ & 0,956 & & & & \\
\hline & EF3 & 0,929 & & & & \\
\hline & EF4 & 0,950 & & & & \\
\hline & EF5 & 0,942 & & & & \\
\hline & EF6 & 0,944 & & & & \\
\hline
\end{tabular}

Fuente: Elaboración Propia

Para la evaluación de la validez discriminante se aplica el criterio Fornell-Larcker, que para lograr la validez discriminante, la raíz cuadrada del AVE de un constructo debe ser mayor que la correlación que éste tenga con cualquier otro constructo (Fornell \& Larcker, 1981) y la relación de correlaciones heterotrait-monotrait (HTMT) (Henseler, Ringle, et al., 2016), cuyos valores deben ser significa- tivamente menores que uno y como umbral valores de 0,85 o 0,9 para indicar pruebas suficientes de validez discriminante (Henseler, Ringle, \& Sarstedt, 2015). En las tablas 2 y 3 se muestran los resultados del criterio Fornell-Larcker y HTMT, de acuerdo con los resultados, se concluye que la validez discriminante está asegurada.

Tabla 2. Evaluación de la validez discriminante de los constructos modelados (criterio de Fornell-Larcker).

\begin{tabular}{|c|c|c|c|c|c|}
\hline Desempeño & 0,944 & & & & \\
\hline Cooperación Comercial & 0,489 & 0,514 & 0,751 & & \\
\hline Cooperación Académica & 0,272 & 0,317 & 0,489 & 0,647 & 0,776 \\
\hline
\end{tabular}

Nota: las variaciones medias extraídas (AVE) se muestran en diagonal en negrita.

Fuente: Elaboración Propia 
Tabla 3. Evaluación de la validez discriminante de los constructos modelados (HTMT).

\begin{tabular}{|c|c|c|c|c|c|}
\hline & Desempeño & Innovación Tecnológica & Cooperación Comercial & Cooperación Profesional & Cooperación Académica \\
\hline \multicolumn{6}{|l|}{ Desempeño } \\
\hline Innovación Tecnológica & 0,900 & & & & \\
\hline Cooperación Comercial & 0,567 & 0,706 & & & \\
\hline Cooperación Profesional & 0,607 & 0,780 & 0,894 & & \\
\hline Cooperación Académica & 0,322 & 0,440 & 0,682 & 0,862 & \\
\hline
\end{tabular}

Fuente: Elaboración Propia

Para evaluar el modelo estructural, deben ser consideradas la valoración de posibles problemas de colinealidad en el modelo estructural, la evaluación del signo algebraico, magnitud y significación estadística de los coeficientes path, valoración del coeficiente de determinación (R2) y valoración de los tamaños de los efectos (f2) (Ali, Rasoolimanesh, Sarstedt, Ringle, \& Ryu, 2018). De acuerdo con (Hair Jr., Sarstedt, Hopkins, \& Kuppelwieser, 2014) existirán indicios de multicolinealidad si valor FIV del modelo estructural son mayores que 5. El valor R2 representa una medida del poder predictivo e indica la cantidad de varianza en el constructo en cuestión, que se explica por sus variables antecedentes en el modelo (Roldán, Sánchez-Franco, \& Real, 2017), los valores de R2 deben ser lo suficientemente altos para que el modelo alcance un nivel mínimo de poder explicativo, como mínimo mayor o igual a 0,10 (Frank \& Miller, 1992). Las estimaciones del coeficiente path debe ser estadísticamente significativo en función del intervalo de confianza del percentil de arranque y su signo debe ser coherente con la hipótesis planteada (Rasoolimanesh, Md Noor, Schuberth, \& Jaafar, 2019).

Los valores R2 significativo para innovación tecnológica $(33,7 \%)$ y para desempeño $(56,8 \%)$. En la tabla 5 se presentan los resultados para el modelo estructural que respalda la aceptación o rechazo de las hipótesis. La figura 2 presenta el modelo en estudio, se ha calculado el coeficiente Stone-Geisser Q2 (Gefen et al., 2000), utilizando el procedimiento blindfolding para medir la relevancia predictiva de las variables latentes endógenas, y se ha obtenido un valor de 0,164 para innovación tecnológica y 0,504 para desempeño, como criterio de evaluación, se deben obtener valores mayores a cero y un modelo perfecto tendría Q2 = 1 (Hair Jr. et al., 2014).

Tabla 5. Resultados del Modelo Estructural. Nota: ${ }^{* \star}$ Significativa $\mathrm{p}<0,001$

\begin{tabular}{|c|c|c|c|c|c|}
\hline \multicolumn{2}{|c|}{ Hipótesis } & \multirow{2}{*}{$\begin{array}{l}\text { Efecto Directo } \\
-0,040\end{array}$} & \multirow{2}{*}{$\frac{\text { t-value }}{1,272}$} & \multirow{2}{*}{$\begin{array}{l}\text { Intervalo de Confianza } \\
\text { (Percentile Bootsrap) }\end{array}$} & \multirow{2}{*}{$\begin{array}{l}\text { Hipótesis } \\
\text { Rechaza }\end{array}$} \\
\hline H1 & Cooperación Académica - Innovación Tecnológica & & & & \\
\hline $\mathrm{H} 3$ & Cooperación Profesional - Innovación Tecnológica & 0,409 & 11,100 & {$[0,347-0,469]$} & Aprueba $^{* * *}$ \\
\hline $\mathrm{H} 4$ & Innovación Tecnológica - Desempeño & 0,790 & 84,272 & {$[0,776-0,807]$} & Aprueba $^{* * *}$ \\
\hline
\end{tabular}

Fuente: Elaboración Propia

Figura 2. Modelo en estudio y resultados

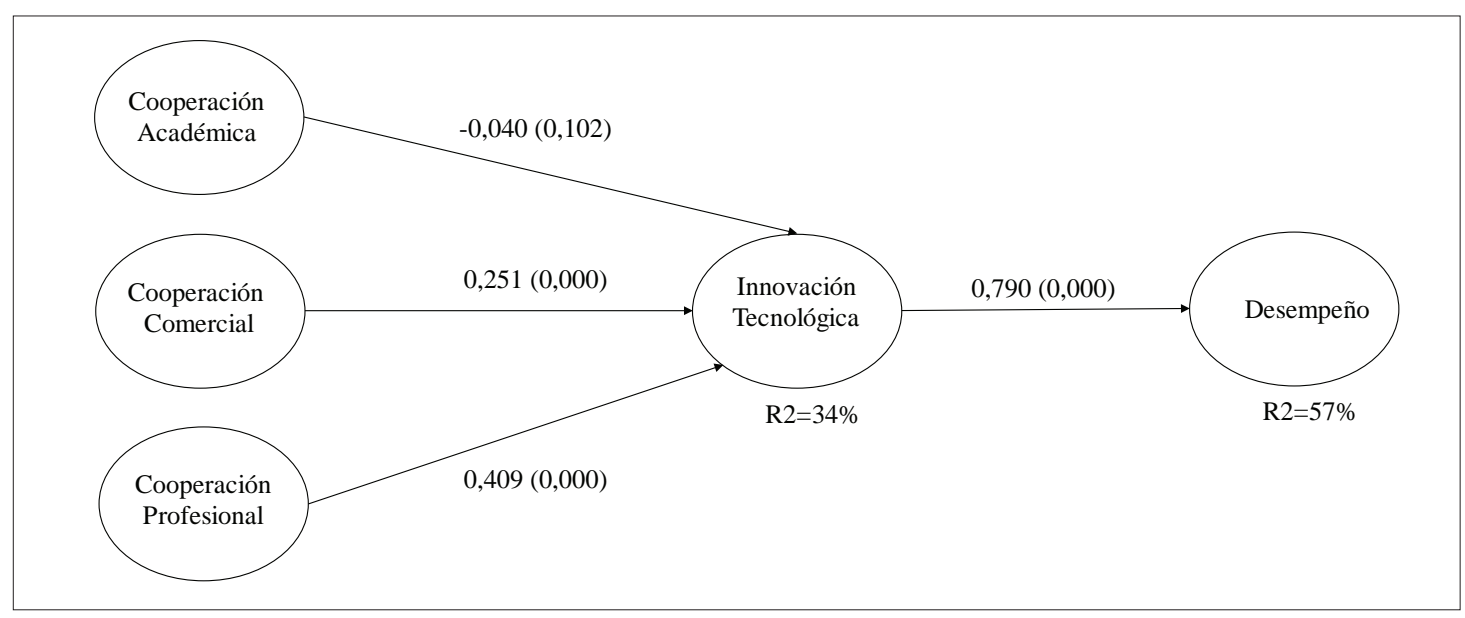

Fuente: Elaboración Propia 


\section{Discusión}

Se analizaron empíricamente las relaciones entre tres tipos de cooperación y el desarrollo de innovación tecnológica en la PYME, así como el impacto que esta tiene en el desempeño, según datos de PYMES chilenas. Los resultados mostraron que tanto la cooperación comercial como la cooperación profesional tuvieron efectos positivos significativos en el desarrollo de innovación tecnológica en empresas pequeñas y medianas, cuestión que está en línea con los argumentos de la literatura, como por ejemplo, (Bengtsson \& Raza-Ullah, 2016; Bolívar-Cruz et al., 2017; Resende et al., 2018). Particularmente considerando el papel de los diferentes actores de los ecosistemas de innovación en Chile, los resultados parecieran estar alineados con los diferentes instrumentos que el estado impulsa, programas que buscan generar proyectos que permitan sistematizar la gestión con apoyo de una entidad experta o el poyo para la implementación de centros que contribuyan a mejorar la productividad de las empresas de menor tamaño, fortalecimiento de capacidades para innovar y la vinculación con el ecosistema. Las políticas gubernamentales actuales son en gran medida propicias para mejorar la vinculación con la PYME y la cooperación con clientes, proveedores, competidores y externos.

Una PYME centrada en la cooperación con proveedores, competidores, clientes o profesional conducirá a elevar sus resultados de innovación tecnológica. Entonces, es clave para este tipo de empresas mantener redes de cooperación, pero debe tenerse en cuenta que dicha relación debe establecer con claridad el tipo de modelo de cooperación, pues tal como señala (Zeng et al., 2010) la cooperación exitosa dependerá del tipo de cooperación definida.

Los hallazgos respecto a la cooperación académica y el impacto en la innovación tecnológica no son aceptados. Hay algunas explicaciones posibles para este resultado. Primero, las políticas de las instituciones académicas aún no se encuentran alineadas con el desarrollo de cooperación con empresas, la gran mayoría de las instituciones de investigación centran sus objetivos y metas en la construcción de conocimiento, y se le otorga poco valor a la cooperación o vinculación en la carrera académica. Esta dificultad, puede impedir que se mejore la relación entre las universidades y los centros de investigación con las empresas PYME. En segundo lugar, un número importante de instituciones académicas o de investigación no ha definido políticas de propiedad intelectual para sus investigadores, este escenario, inhibe la participación de estos en el desarrollo de innovación tecnológica, y en los casos en que ocurre su participación se realiza de forma profesional y no institucional. Dado que en general las empresas cooperan con Universidades y centros de investigación para acceder a conocimientos científicos, técnicos o nuevas tecnologías (Barroso Simao, Gouveia Rodrigues, \& Madeira, 2016), se debieran potenciar programas gubernamentales que potencien dicha relación.

Nuestro trabajo evidencia empíricamente la relación sobre innovación y desempeño en las empresas pequeñas y medianas chilenas, dicha evidencia es importante, pues esta relación positiva se ha indicado que depende del contexto (Rosenbusch, Brinckmann, \& Bausch, 2011). Las empresas grandes tienen mayores probabilidades de invertir y participar en innovación, dicha capacidad disminuye con empresas pequeñas y medianas (Hashi \& Nebojsa, 2013), y nuestra contribución está en línea con lo reportado para países emergentes (Santi \& Santoleri, 2017).

\section{Conclusiones}

De acuerdo con los resultados de la décima encuesta de innovación, un $19.3 \%$ de las empresas que innovan, dice haber participado en acciones de cooperación en proyectos de innovación. Por ello, el objetivo de este estudio fue analizar los efectos de diferentes actividades de cooperación sobre la innovación tecnológica y sus efectos en el desempeño de empresas en Chile. Los resultados indican que (a) las empresas PYME que colaboran con clientes, proveedores, competidores y/o consultores presentan mejores resultados de innovación tecnológica; (b) las empresas PYME que participan en actividades de colaboración a nivel profesional e industrial, en conferencias, ferias, exposiciones y/o con profesionales al interior de las organizaciones presentan mejores resultados de innovación tecnológica y (c) el desempeño de las empresas PYME se ve afectado positivamente por el desarrollo de innovaciones tecnológicas.

Esto se puede explicar por los siguientes antecedentes en el caso de Chile, el tamaño de la empresa está relacionado con la probabilidad de que las empresas se dediquen a actividades de innovación (Benavente, 2007) y se ha sugerido que existen sinergias sin explotar entre las políticas de apoyo a la innovación y las políticas de apoyo al espíritu empresarial en el contexto de las iniciativas de desarrollo regional (Modrego, Mccann, Foster, \& Olfert, 2015), las políticas públicas deben promover acciones para mejorar la relación entre las empresas y sus redes no relacionadas (Basco \& Calabrò, 2016), dado que se ha propuesto que las empresas chilenas funcionan en un contexto de individualismo, lo que tiene un efecto negativo en la relación entre la innovación y el rendimiento (Rosenbusch et al., 2011).

Este estudio hace una contribución metodológica significativa a la literatura de innovación, al aplicar un análisis mediante Smart-PLS, y los hallazgos tienen una serie de implicaciones prácticas, tanto para para las empresas como para los gobiernos. Los resultados se encuentran en línea con el llamado a que las políticas públicas desarrollen programas para promover redes de innovación para ayudar a las empresas a generar innovaciones (Geldes, Felzensztein, \& Palacios-Fenech, 2017). De acuerdo a nuestros resultados, se debiera propiciar una mayor cooperación entre las entidades académicas con las empresas para fortalecer la innovación tecnológica, tal como se ha planteado ampliamente (Barrie, Zawdie, \& João, 2019; W. Liu \& Atuahene-Gima, 2018; Ueasangkomsate \& Jangkot, 2018).

Desde el punto de vista de las empresas, la cooperación es una posibilidad de reducir riesgos asociados con los procesos de innovación y compartir gastos (personal especializado, laboratorios, tecnología, estudios) si trabajan en conjunto. Las empresas que participan en actividades basadas en la cooperación estarán expuestas a flujos de conocimiento más densos que las entidades que no cooperan (Raposo, Ferreira, \& Fernandes, 2014), dado que se puede facilitar el desarrollo 
de innovaciones al beneficiarse del acceso a diferentes conocimientos (Serrano-Bedia et al., 2018). Los gerentes de las empresas deben establecer que estrategia seguir para el desempeño de la innovación, pues genera mayores efectos la cooperación con entidades comerciales o profesionales que con instituciones académicas.

Las empresas que desarrollan innovación tecnológica tienen un impacto positivo y significativo en el desempeño de las empresas, que se ven reflejadas en la ampliación de la gama de bienes y servicios, ingreso a nuevos mercados o incrementos de la participación en el mercado actual, mejora en la calidad de los bienes y servicios, aumento de la capacidad y/o flexibilidad para la producción de bienes y servicios, reducción de costos y/o reducción de impacto medioambiental o mejorar la sanidad y la seguridad. Considerando que en Chile, un número importante de empresas PYME posee una concentración de clientes, el desarrollar innovación tecnológica tendrá efecto en el desempeño de la misma, y disminuir el riesgo de incertidumbre al concentrar en un número reducido de clientes.

Los gobiernos deben promover interacciones con las empresas, lo que puede aumentar las posibilidades de mayor desarrollo de innovación en las empresas (Stubrin, 2017) y las políticas públicas debieran abordar este desafío. Existen diversos obstáculos a la innovación (AcunaOpazo \& Castillo-Vergara, 2018), muchas de ellas se deben a la falta de recursos humanos, financieros y especializados (Iturrioz, Aragón, \& Narvaiza, 2015) y existe la necesidad de una acción política más efectiva para aliviar los obstáculos que disuaden a las empresas de invertir en innovación y proporcionar mejores condiciones comerciales (Crespi \& Zuniga, 2012), que incluyan la cooperación.

Nuestros resultados, no muestran resultados significativos respecto al impacto de la cooperación académica en el desarrollo de innovación en la PYME, aun cuando se ha señalado que las universidades y los institutos de investigación tienen un impacto valioso dentro del proceso de creación de conocimiento e innovación y generalmente ocurren mediante un intercambio explícito de conocimiento (Ben Arfi, Hikkerova, \& Sahut, 2018). Con lo cual, futuras investigaciones podrían abordar si la cooperación académica puede mediar el desarrollo de investigación y desarrollo dentro de las empresas como determinante de la innovación. Dado que el estudio se limitó a la muestra general, futuras investigaciones podrían considerar el análisis por sectores económicos o analizar de forma separada la innovación de producto (radical e incremental) y la innovación de proceso (directas e indirectas) o innovaciones no tecnológicas.

\section{References}

Abdul-Halim, H., Ahmad, N. H., Geare, A., \& Thurasamy, R. (2019). Innovation Culture in SMEs: The Importance of Organizational Culture, Organizational Learning and Market Orientation. Entrepreneurship Research Journal, 9(3), 1-14. https://doi.org/10.1515/erj-2017-0014

Acuna-Opazo, C., \& Castillo-Vergara, M. (2018). Barriers to non-technological innovation : Impact on business performance in an emerging economy. Contaduria y Administración, 63(3), 1-22.
Akdoğan, A. A., \& Cingšz, A. (2012). An Empirical Study on Determining the Attitudes of Small and Medium Sized Businesses (SMEs) Related to Coopetition. Procedia - Social and Behavioral Sciences, 58, 252-258. https://doi.org/10.1016/j.sbspro.2012.09.999

Ali, F., Rasoolimanesh, S. M., Sarstedt, M., Ringle, C. M., \& Ryu, K. (2018). An assessment of the use of partial least squares structural equation modeling (PLS-SEM) in hospitality research. International Journal of Contemporary Hospitality Management, 30(1), 514-538. https://doi.org/10.1108/IJCHM-10-2016-0568

Atalay, M., Anafarta, N., \& Sarvan, F. (2013). The Relationship between Innovation and Firm Performance: An Empirical Evidence from Turkish Automotive Supplier Industry. Procedia - Social and Behavioral Sciences, 75, 226-235. https://doi.org/10.1016/j.sbspro.2013.04.026

Barclay, D., Higgins, C., \& Thompson, R. (1995). The Partial Least Squares (PLS) Approach to Causal Modeling: Personal computer adoption and usa as an ilustration. Technology Studies, 2(2), 285-309.

Barrie, J., Zawdie, G., \& João, E. (2019). Assessing the role of triple helix system intermediaries in nurturing an industrial biotechnology innovation network. Journal of Cleaner Production, 214, 209-223. https://doi.org/10.1016/j.jclepro.2018.12.287

Barroso, C., Cepeda Carrión, G., \& Roldán, J. L. (2010). Handbook of Partial Least Squares. In Handbook of Partial Least Squares (pp. 427-447). https://doi.org/10.1007/978-3-540-32827-8

Barroso Simao, L., Gouveia Rodrigues, R., \& Madeira, M. J. (2016). External relationships in the organizational innovation. RAI Revista de Administração e Inovação, 13(3), 156-165. https://doi. org/10.1016/j.rai.2016.06.002

Basco, R., \& Calabrò, A. (2016). Open innovation search strategies in family and non-family SMEs. Academia Revista Latinoamericana de Administración, 29(3), 279-302. https://doi.org/10.1108/ARLA-07-2015-0188

Ben Arfi, W., Hikkerova, L., \& Sahut, J. M. (2018). External knowledge sources, green innovation and performance. Technological Forecasting and Social Change, 129(January), 210-220. https://doi.org/10.1016/j. techfore.2017.09.017

Benavente, J. M. (2007). The role of research and innovation in promoting productivity in chile. Economics of Innovation and New Technology, 15(4-5), 301-315.

Bengtsson, M., \& Raza-Ullah, T. (2016). A systematic review of research on coopetition: Toward a multilevel understanding. Industrial Marketing Management, 57, 23-39. https://doi.org/10.1016/j.indmarman.2016.05.003

Bolívar-Cruz, A., Fernández-Monroy, M., \& Galván-Sánchez, I. (2017). La cooperación tecnológica universidad-empresa: El rol de la comunicación. Journal of Technology Management and Innovation, 12(3), 67-77. 
Casanova, L., Cornelius, P. K., \& Dutta, S. (2017). Global Innovation Competitiveness: How Emerging Economies Compare ${ }^{*}{ }^{*}$ The contributions of Rafael Reynoso, Jordan Litner, Bruno Lanvin, Sacha WunschVincent, and Francesca Guadagno are gratefully acknowledged. Financing Entrepreneurship and Innovation in Emerging Markets. https:// doi.org/10.1016/b978-0-12-804025-6.00002-2

Castillo-Vergara, M., Barrios Galleguillos, N., Jofré Cuello, L., Alvarez-Marin, A., \& Acuña-Opazo, C. (2018). Does socioeconomic status influence student creativity? Thinking Skills and Creativity, 29(May), 142-152. https://doi.org/10.1016/j.tsc.2018.07.005

Cepeda Carrión, G., \& Roldán Salgueiro, J. L. (2004). Aplicando en la práctica la técnica PLS en la administración de empresas.

Chin, W. W. (1998). The Partial Least Squares Approach to Structural Equation Modeling. In The Partial Least Squares Approach to Structural Equation Modeling (pp. 295-336).

Chin, W. W. (2010). Handbook of Partial Least Squares. How to Write Up and Report PLS Analyses. https://doi.org/10.1007/978-3-540-32827-8

Chou, H. H., \& Zolkiewski, J. (2018). Coopetition and value creation and appropriation: The role of interdependencies, tensions and harmony. Industrial Marketing Management, 70(August 2017), 25-33. https://doi.org/10.1016/j.indmarman.2017.08.014

CORFO. (2013). Memoria Corfo 2010-2013.

Crespell, P., \& Hansen, E. (2008). Work climate, innovativeness, and firm performance in the US forest sector : in search of a conceptual framework. Canadian Journal of Forest Research, 38(7), 1703-1715. https://doi.org/10.1139/X08-027

Crespi, G., \& Zuniga, P. (2012). Innovation and Productivity: Evidence from Six Latin American Countries. World Development, 40(2), 273-290. https://doi.org/10.1016/j.worlddev.2011.07.010

Dorn, S., Schweiger, B., \& Albers, S. (2016). Levels, phases and themes of coopetition: A systematic literature review and research agenda. European Management Journal, 34(5), 484-500. https://doi. org/10.1016/j.emj.2016.02.009

Edwards-Schachter, M. (2018). The nature and variety of innovation. International Journal of Innovation Studies, 2(2), 65-79. https://doi. org/10.1016/j.ijis.2018.08.004

Ferreira, J., Coelho, A., \& Moutinho, L. (2018). Technovation Dynamic capabilities, creativity and innovation capability and their impact on competitive advantage and fi $\mathrm{rm}$ performance: The moderating role of entrepreneurial orientation. Technovation, (Febrero 2018), 1-18. https://doi.org/10.1016/j.technovation.2018.11.004

Fornell, C., \& Larcker, D. F. (1981). Evaluating Structural Equation Models with Unobservable Variables and Measurement Error. Journal of Marketing Research, 18(1), 39-50. https://doi.org/10.20546/ ijcrar.2016.409.006
Fossas-olalla, M., Minguela-rata, B., \& Fernández-menéndez, J. (2015). Product innovation: When should suppliers begin to collaborate? Journal of Business Research, 68(7), 1404-1406. https://doi. org/10.1016/j.jbusres.2015.01.022

Frank, F. R., \& Miller, N. B. (1992). A primer for soft modeling. https:// doi.org/10.1002/pro.5560050910

Gefen, D., Straub, D. W., \& Boudreau, M.-C. (2000). Structural Equation Modeling and Regression: Guidelines for Research Practice. Communications of the Association for Information Systems, 4(August), 1-79. https://doi.org/10.17705/1CAIS.00407

Geldes, C., \& Felzensztein, C. (2013). Academia Revista Latinoamericana de Administración Marketing innovations in the agribusiness sector Article information: Academia Revista Latinoamericana de Administración, 26(1), 108-138. https://doi.org/10.1108/ARLA-092015-0253

Geldes, C., Felzensztein, C., \& Palacios-Fenech, J. (2017). Technologi$\mathrm{cal}$ and non-technological innovations, performance and propensity to innovate across industriesThe case of an emerging economy. Industrial Marketing Management, 61, 55-66. https://doi.org/10.1016/j. indmarman.2016.10.010

Genc, E., Dayan, M., \& Genc, O. F. (2019). The impact of SME internationalization on innovation: The mediating role of market and entrepreneurial orientation. Industrial Marketing Management, (December 2018), 0-1. https://doi.org/10.1016/j.indmarman.2019.01.008

Giannopoulou, E., Barlatier, P. J., \& Pénin, J. (2019). Same but different? Research and technology organizations, universities and the innovation activities of firms. Research Policy, 48(1), 223-233. https:// doi.org/10.1016/j.respol.2018.08.008

Gnyawali, D. R., \& Park, B. J. (2011). Co-opetition between giants: Collaboration with competitors for technological innovation. Research Policy, 40(5), 650-663. https://doi.org/10.1016/j.respol.2011.01.009

Guimón, J., Chaminade, C., Maggi, C., \& Salazar-Elena, J. C. (2018). Policies to Attract R\&D-related FDI in Small Emerging Countries: Aligning Incentives With Local Linkages and Absorptive Capacities in Chile. Journal of International Management, 24(2), 165-178. https://doi.org/10.1016/j.intman.2017.09.005

Gunday, G., Ulusoy, G., Kilic, K., \& Alpkan, L. (2011). Effects of innovation types on firm performance. International Journal of Production Economics, 133(2), 662-676. https://doi.org/10.1016/j. ijpe.2011.05.014

Hair, J. F., Hult, G. T. M., Ringle, C., \& Sarstedt, M. (2017). A primer on partial least squares structural equation modeling (PLS-SEM). Sage Publications.

Hair Jr., J., Sarstedt, M., Hopkins, L., \& Kuppelwieser, G. V. (2014). Partial least squares structural equation modeling (PLS-SEM). European Business Review, 26(2), 106-121. https://doi.org/10.1108/ebr-10-2013-0128 
Hashi, I., \& Nebojsa, S. (2013). The impact of innovation activities on firm performance using a multi-stage model: Evidence from the Community Innovation Survey 4. Research Policy, 42, 353-366. https://doi.org/10.1016/j.respol.2012.09.011

Heidenreich, S., Landsperger, J., \& Spieth, P. (2016). Are Innovation Networks in Need of a Conductor? Examining the Contribution of Network Managers in Low and High Complexity Settings. Long Range Planning, 49(1), 55-71. https://doi.org/10.1016/j.lrp.2014.03.003

Henseler, J. (2016). Some inconvenient truths about PLS path modeling. 9th International Conference on PLS and Related Methods (PLS'16), (June), 74-76.

Henseler, J. (2018). Partial least squares path modeling: Quo vadis? Quality and Quantity, 52(1). https://doi.org/10.1007/s11135-018-0689-6

Henseler, J., Hubona, G., \& Ray, P. A. (2016). Using PLS path modeling in new technology research: Updated guidelines. Industrial $\mathrm{Ma}$ nagement and Data Systems, 116(1), 2-20. https://doi.org/10.1108/ IMDS-09-2015-0382

Henseler, J., Ringle, C. M., \& Sarstedt, M. (2015). A new criterion for assessing discriminant validity in variance-based structural equation modeling. Journal of the Academy of Marketing Science, 43(1), 115135. https://doi.org/10.1007/s11747-014-0403-8

Henseler, J., Ringle, C. M., \& Sarstedt, M. (2016). Testing measurement invariance of composites using partial least squares. International Marketing Review, 33(3), 405-431. https://doi.org/10.1108/IMR09-2014-0304

Heredia, J. A., Geldes, C., Kunc, M. H., \& Flores, A. (2019). Technovation New approach to the innovation process in emerging economies: The manufacturing sector case in Chile and Peru. Technovation, 79(March 2016), 35-55. https://doi.org/10.1016/j.technovation.2018.02.012

Heredia Pérez, J. A., Geldes, C., Kunc, M. H., \& Flores, A. (2019). New approach to the innovation process in emerging economies: The manufacturing sector case in Chile and Peru. Technovation, 79(March 2018), 35-55. https://doi.org/10.1016/j.technovation.2018.02.012

Höflinger, P. J., Nagel, C., \& Sandner, P. (2017). Reputation for technological innovation: Does it actually cohere with innovative activity? Journal of Innovation \& Knowledge, 3(1), 26-39. https://doi. org/10.1016/j.jik.2017.08.002

Howell, A. (2017). Picking 'winners" in China: Do subsidies matter for indigenous innovation and firm productivity?' China Economic Review, 44(71603009), 154-165. https://doi.org/10.1016/j.chieco.2017.04.005

Hu, L. T., \& Bentler, P. M. (1999). Cutoff criteria for fit indexes in covariance structure analysis: Conventional criteria versus new alternatives. Structural Equation Modeling, 6(1), 1-55. https://doi. org/10.1080/10705519909540118
Hughes, P., Souchon, A. L., Nemkova, E., Hodgkinson, I. R., Oliveira, J. S., Boso, N., ... Sy-Changco, J. (2018). Quadratic effects of dynamic decision-making capability on innovation orientation and performance: Evidence from Chinese exporters. Industrial Marketing Management, (October 2017), 1-11. https://doi.org/10.1016/j.indmarman.2018.11.005

Instituto Nacional de Estadisticas. (2018). Décima Encuesta de Innovación en Empresas 2015-2016.

Intarakumnerd, P., \& Goto, A. (2018). Role of public research institutes in national innovation systems in industrialized countries: The cases of Fraunhofer, NIST, CSIRO, AIST, and ITRI. Research Policy, 47(7), 1309-1320. https://doi.org/10.1016/j.respol.2018.04.011

Iturrioz, C., Aragón, C., \& Narvaiza, L. (2015). How to foster shared innovation within SMEs ' networks : Social capital and the role of intermediaries. European Management Journal, 33(2), 104-115. https:// doi.org/10.1016/j.emj.2014.09.003

Jia, J., \& Ma, G. (2017). Do R\&D tax incentives work? Firm-level evidence from China. China Economic Review, 46(August), 50-66. https://doi.org/10.1016/j.chieco.2017.08.012

Karabulut, A. T. (2015). Effects of Innovation Types on Performance of Manufacturing Firms in Turkey. Procedia - Social and Behavioral Sciences, 195, 1355-1364. https://doi.org/10.1016/j.sbspro.2015.06.322

Klerkx, L., Álvarez, R., \& Campusano, R. (2015). The emergence and functioning of innovation intermediaries in maturing innovation systems: the case of Chile. Innovation and Development, 5(1), 73-91. https://doi.org/10.1080/2157930X.2014.921268

Laforet, S. (2013). Organizational innovation outcomes in SMEs: Effects of age, size, and sector. Journal of World Business, 48(4), 490502. https://doi.org/10.1016/j.jwb.2012.09.005

Lafuente, E., Leiva, J. C., Moreno-Gómez, J., \& Szerb, L. (2019). A non-parametric analysis of competitiveness efficiency: The relevance of firm size and the configuration of competitive pillars. BRQ Business Research Quarterly. https://doi.org/10.1016/j.brq.2019.02.002

Lavoie, C., Lavoie, C., Abdulnour, G., Lavoie, C., \& Abdulnour, G. (2015). ScienceDirect define a a creative creative SME How to to define define a a creative creative SME creative. IFAC-PapersOnLine, 48(3), 910-915. https://doi.org/10.1016/j.ifacol.2015.06.199

Lin, J. Y. (2017). Balancing industry collaboration and academic innovation: The contingent role of collaboration-specific attributes. Technological Forecasting and Social Change, 123, 216-228. https://doi. org/10.1016/j.techfore.2016.03.016

Lindström, T., \& Polsa, P. (2016). Coopetition close to the customer - A case study of a small business network. Industrial Marketing Management, 53, 207-215. https://doi.org/10.1016/j.indmarman.2015.06.005 
Liu, D., Chen, T., Liu, X., \& Yu, Y. (2018). Do more subsidies promote greater innovation? Evidence from the Chinese electronic manufacturing industry. Economic Modelling, (November). https://doi. org/10.1016/j.econmod.2018.11.027

Liu, W., \& Atuahene-Gima, K. (2018). Enhancing product innovation performance in a dysfunctional competitive environment: The roles of competitive strategies and market-based assets. Industrial Marketing Management, 73(December 2016), 7-20. https://doi. org/10.1016/j.indmarman.2018.01.006

Luo, L., Yang, Y., Luo, Y., \& Liu, C. (2016). Export, subsidy and innovation: China's state-owned enterprises versus privately-owned enterprises. Economic and Political Studies, 4(2), 137-155. https://doi.org/1 $0.1080 / 20954816.2016 .1180766$

Martelo-Landroguez, S., Cegarra Navarro, J. G., \& Cepeda-Carrión, G. (2019). Uncontrolled counter-knowledge: its effects on knowledge management corridors. Knowledge Management Research and Practice, 00(00), 1-10. https://doi.org/10.1080/147782 38.2019.1599497

Modrego, F., Mccann, P., Foster, W. E., \& Olfert, M. R. (2015). Regional entrepreneurship and innovation in Chile : a knowledge matching approach. Small Business Economics, 44, 685-703. https://doi. org/10.1007/s11187-014-9612-2

Müller, T., Schuberth, F., \& Henseler, J. (2018). PLS path modeling - a confirmatory approach to study tourism technology and tourist behavior. Journal of Hospitality and Tourism Technology, 9(3), 249-266. https://doi.org/10.1108/JHTT-09-2017-0106

OECD/European Communities. (2005). Oslo manual: Guidelines for collecting and interpreting innovation data. Publications de l'OCDE.

Park, B. J. R., Srivastava, M. K., \& Gnyawali, D. R. (2014). Walking the tight rope of coopetition: Impact of competition and cooperation intensities and balance on firm innovation performance. Industrial Marketing Management, 43(2), 210-221. https://doi.org/10.1016/j. indmarman.2013.11.003

Perkins, G., Lean, J., \& Newbery, R. (2017). The Role of Organizational Vision in Guiding Idea Generation within SME Contexts. Creativity and Innovation Management, 26(1), 75-90. https://doi. org/10.1111/caim.12206

Radziwon, A., \& Bogers, M. (2018). Open innovation in SMEs: Exploring inter-organizational relationships in an ecosystem. Technological Forecasting and Social Change. https://doi.org/https://doi. org/10.1016/j.techfore.2018.04.021

Rajapathirana, R. P. J., \& Hui, Y. (2017). Relationship between innovation capability, innovation type, and firm performance. Journal of Innovation \& Knowledge, 3(1), 44-55. https://doi.org/10.1016/j. jik.2017.06.002
Raposo, M. L., Ferreira, J. J. M., \& Fernandes, C. I. (2014). Local and cross-border SME cooperation: Effects on innovation and performance. Revista Europea de Dirección y Economía de La Empresa, 23(4), 157-165. https://doi.org/10.1016/j.redee.2014.08.001

Rasoolimanesh, S. M., Md Noor, S., Schuberth, F., \& Jaafar, M. (2019). Investigating the effects of tourist engagement on satisfaction and loyalty. Service Industries Journal, 0(0), 1-16. https://doi.org/10.1080/0 2642069.2019.1570152

Reinartz, W., Haenlein, M., \& Henseler, J. (2009). An empirical comparison of the efficacy of covariance-based and variance-based SEM. International Journal of Research in Marketing, 26(4), 332-344. https://doi.org/10.1016/j.ijresmar.2009.08.001

Resende, L. M. M. de, Volski, I., Betim, L. M., Carvalho, G. D. G. de, Barros, R. de, \& Senger, F. P. (2018). Critical success factors in coopetition: Evidence on a business network. Industrial Marketing Management, 68(November 2017), 177-187. https://doi.org/10.1016/j. indmarman.2017.10.013

Ringle, C. M., Wende, S., \& Becker, J. M. (2017). SmartPLS-Statistical Software For Structural Equation Modeling.

Rojas, M. G. A., Solis, E. R. R., \& Zhu, J. J. J. (2018). Innovation and network multiplexity: R\&D and the concurrent effects of two collaboration networks in an emerging economy. Research Policy, 47(6), 1111-1124. https://doi.org/10.1016/j.respol.2018.03.018

Roldán, J. L., Sánchez-Franco, M. J., \& Real, J. C. (2017). From frequency of use to social integration: The mediation of routinization and infusion in Tuenti community. European Research on Management and Business Economics, 23(2), 63-69. https://doi.org/10.1016/j. iedeen.2016.07.002

Rosenbusch, N., Brinckmann, J., \& Bausch, A. (2011). Journal of Business Venturing Is innovation always bene fi cial ? A meta-analysis of the relationship between innovation and performance in SMEs. Journal of Business Venturing, 26(4), 441-457. https://doi.org/10.1016/j. jbusvent.2009.12.002

Santi, C., \& Santoleri, P. (2017). Exploring the link between innovation and growth in Chilean firms. Sma, 49, 445-467. https://doi. org/10.1007/s11187-016-9836-4

Sarooghi, H., Libaers, D., \& Burkemper, A. (2015). Examining the relationship between creativity and innovation: A meta-analysis of organizational, cultural, and environmental factors. Journal of Business Venturing, 30(5), 714-731. https://doi.org/https://doi.org/10.1016/j. jbusvent.2014.12.00

Scandura, A. (2016). University-industry collaboration and firms' R\&D effort. Research Policy, 45(9), 1907-1922. https://doi. org/10.1016/j.respol.2016.06.009 
Schiavone, F., \& Simoni, M. (2011). An experience-based view of coopetition in R\&D networks. European Journal of Innovation Management, 14(2), 136-154.

Serrano-Bedia, A. M., López-Fernández, M. C., \& García-Piqueres, G. (2018). Complementarity between innovation knowledge sources: Does the innovation performance measure matter? BRQ $\mathrm{Bu}$ siness Research Quarterly, 21(1), 53-67. https://doi.org/10.1016/j. brq.2017.09.001

Sosik, J. J., Kahai, S. S., \& Piovoso, M. J. (2009). Silver Bullet or Voodoo Statistics? Group \& Organization Management, 34(1), 5-36. https://doi.org/10.1177/1059601108329198

Stubrin, L. (2017). Innovation, learning and competence building in the mining industry. The case of knowledge intensive mining suppliers ( KIMS ) in Chile. Resources Policy, 54(October), 167-175. https://doi.org/10.1016/j.resourpol.2017.10.009

Szücs, F. (2018). Research subsidies, industry-university cooperation and innovation. Research Policy, 47(7), 1256-1266. https://doi. org/10.1016/j.respol.2018.04.009

Tello Gamarra, J., Machado Leo, R., Silva Avila, A. M., \& Wendland, J. (2019). Innovation studies in Latin America: a bibliometric analysis. Journal of Technology Management \& Innovation, 13(4), 24-36. https://doi.org/10.4067/s0718-27242018000400024

Torregrosa-Hetland, S., Pelkonen, A., Oksanen, J., \& Kander, A. (2019). The prevalence of publicly stimulated innovations -A comparison of Finland and Sweden, 1970-2013. Research Policy, (December 2018), 1-12. https://doi.org/10.1016/j.respol.2019.02.001

Trigo, A. (2011). Innovación y la importancia de los flujos externos de información: Una propuesta para el análisis de los contactos informales. Journal of Technology Management and Innovation, 6(2), 62-75.
Ueasangkomsate, P., \& Jangkot, A. (2018). Enhancing the innovation of small and medium enterprises in food manufacturing through Triple Helix Agents. Kasetsart Journal of Social Sciences, 1-9. https://doi. org/10.1016/j.kjss.2017.12.007

Vivas, C., \& Barge-Gil, A. (2015). Impact on firms of the use of knowledge external sources: A systematic review of the literature. Journal of Economic Surveys, 29(5), 943-964. https://doi.org/10.1111/joes.12089

Weber, B., \& Heidenreich, S. (2018). When and with whom to cooperate? Investigating effects of cooperation stage and type on innovation capabilities and success. Long Range Planning, 51(2), 334-350. https://doi.org/10.1016/j.lrp.2017.07.003

Williams, L. J., Vandenberg, R. J., \& Edwards, J. R. (2009). 12 structural equation modeling in management research: A guide for improved analysis. Academy of Management Annals, 3(1), 543-604.

Wold, H. (1984). Systems Analysis by Partial Least Squares. In Measuring the Unmeasurable (pp. 221-252). https://doi.org/10.1016/j. acra.2010.05.001

Wu, J. (2014). Cooperation with competitors and product innovation: Moderating effects of technological capability and alliances with universities. Industrial Marketing Management, 43(2), 199-209. https:// doi.org/10.1016/j.indmarman.2013.11.002

Xu, L., Peng, X., \& Prybutok, V. (2019). Formative measurements in operations management research: Using partial least squares. Quality Management Journal, 26(1), 18-31. https://doi.org/10.1080/1068696 7.2018.1542287

Zeng, S. X., Xie, X. M., \& Tam, C. M. (2010). Relationship between cooperation networks and innovation performance of SMEs. Technovation, 30(3), 181-194. https://doi.org/10.1016/j.technovation.2009.08.003 
J. Technol. Manag. Innov. 2019. Volume 14, Issue 4 